\title{
Identification of duck liver-expressed antimicrobial peptide 2 and characterization of its bactericidal activity
}

\author{
Yeojin Hong ${ }^{1}$, Anh Duc Truong ${ }^{1,2}$, Janggeun Lee ${ }^{1}, K_{y u n g b a e k ~ L e e}{ }^{1}$, Geun-Bae Kim ${ }^{1}, K_{\text {Kang-Nyeong Heo }}{ }^{\text {, }}$ \\ Hyun S. Lillehoj ${ }^{4}$, and Yeong Ho Hong,*
}

\author{
* Corresponding Author: Yeong Ho Hong \\ Tel: +82-31-670-3025, Fax: +82-31-671-3025 \\ E-mail: yhong@cau.ac.kr \\ 1 Department of Animal Science and Technology, \\ Chung-Ang University, Anseong 17546, Korea \\ 2 Department of Biochemistry and Immunology, \\ National Institute of Veterinary Research, Dong Da, \\ Hanoi 10000, Vietnam \\ ${ }^{3}$ Poultry Research Institute, National Institute of \\ Animal Science, RDA, Pyeongchang 25342, Korea \\ ${ }^{4}$ Animal Biosciences and Biotechnology Laboratory, \\ Agricultural Research Services, United States \\ Department of Agriculture, Beltsville, MD 20705, USA \\ ORCID \\ Yeojin Hong \\ https://orcid.org/0000-0001-8071-8183 \\ Anh Duc Truong \\ https://orcid.org/0000-0002-2472-8165 \\ Janggeun Lee \\ https://orcid.org/0000-0002-8552-9581 \\ Kyungbaek Lee \\ https://orcid.org/0000-0002-7887-2437 \\ Geun-Bae Kim \\ https://orcid.org/0000-0001-8531-1104 \\ Kang-Nyeong Heo \\ https://orcid.org/0000-0002-2757-4333 \\ Hyun S. Lillehoj \\ https://orcid.org/0000-0001-7755-6216 \\ Yeong Ho Hong \\ https://orcid.org/0000-0002-4510-7851
}

Submitted Jul 28, 2018; Revised Oct 9, 2018; Accepted Oct 26, 2018
Objective: This study was conducted to identify duck liver-expressed antimicrobial peptide 2 (LEAP-2) and demonstrate its antimicrobial activity against various pathogens.

Methods: Tissue samples were collected from 6 to 8-week-old Pekin ducks (Anas platyrhynchos domesticus), total RNA was extracted, and CDNA was synthesized. To confirm the duck LEAP-2 transcript expression levels, quantitative real-time polymerase chain reaction was conducted. Two kinds of peptides (a linear peptide and a disulfide-type peptide) were synthesized to compare the antimicrobial activity. Then, antimicrobial activity assay and fluorescence microscopic analysis were conducted to demonstrate duck LEAP-2 bactericidal activity.

Results: The duck LEAP-2 peptide sequence showed high identity with those of other avian species $(>85 \%)$, as well as more than $55 \%$ of identity with mammalian sequences. LEAP-2 mRNA was highly expressed in the liver with duodenum next, and then followed by lung, spleen, bursa and jejunum and was the lowest in the muscle. Both of LEAP-2 peptides efficiently killed bacteria, although the disulfide-type LEAP-2 showed more powerful bactericidal activity. Also, gram-positive bacteria was more susceptible to duck LEAP-2 than gram-negative bacteria. Using microscopy, we confirmed that LEAP-2 peptides could kill bacteria by disrupting the bacterial cell envelope.

Conclusion: Duck LEAP-2 showed its antimicrobial activity against both gram-positive and gram-negative bacteria. Disulfide bonds were important for the powerful killing effect by disrupting the bacterial cell envelope. Therefore, duck LEAP-2 can be used for effective antibiotics alternatives.

Keywords: Antimicrobial Peptides; Liver-expressed Antimicrobial Peptide 2 (LEAP-2); Duck; Disulfide Bond; Pathogens

\section{INTRODUCTION}

With increasing concerns about food and environmental safety with respect to the emergence of antibiotic resistance in pathogens and the increasing presence of antibiotic residues in meat products in the last few decades, novel strategies for alternatives to antibiotics to reduce the usage of antibiotic growth promoters (AGPs) in poultry production are actively under development [1]. In particular, the limited availability of drug alternatives to manage avian diseases poses a major economic challenge in the European Union where AGPs have been banned since 2006.

Antimicrobial peptides (AMPs) are important peptides of innate immune systems. These peptides are composed of 12 to 50 amino acids, which include two or more positively charged residues and a hydrophobic residue [2-4]. In general, AMPs are known to have antimicrobial activity against gram-negative bacteria, gram-positive bacteria, fungi, viruses, parasites, and even cancer cells $[5,6]$. The general target of AMPs is the bacterial cell membrane, although 
some AMPs act to inhibit DNA, RNA, and/or protein synthesis. Other properties of AMPs include the induction of cytokine release, cell proliferation, wound healing, and chemotaxis $[7,8]$. In mammals, AMPs are synthesized primarily by phagocytes or epithelial cells. Conversely, many insect AMPs are produced predominantly by the fat body, the functional equivalent of the liver, and secreted into the hemolymph after septic injury [9-11].

There are various infectious diseases that poultry can take. Avian influenza, avian tuberculosis, fowl cholera, fowl pox, infectious bronchitis, infectious bursal disease, Marek's disease, mycoplasmosis, necrotic enteritis and salmonellosis, etc. are triggered by viruses, parasites and bacteria. The AMPs can be used for poultry infectious disease control because it have antiviral, anti-parasite and antibacterial activities $[5,6]$.

Liver-expressed antimicrobial peptide 2 (LEAP-2) was first isolated from a human in 2003 [12] and has been reported from other species, including mice, cattle, pigs, and chicken [13]. In most species, LEAP-2 is a cysteine-rich and cationic protein $[14,15]$, which generally has a conserved core structure with two disulfide bonds that play a crucial role in bacterial killing [16]. In poultry, LEAP-2 sequences have been characterized in chicken and Japanese quail $[17,18]$, but duck LEAP-2 has not yet been characterized.

Therefore, in this study, we identified and characterized the duck $L E A P$-2 gene to determine its antimicrobial activity against gram-positive and gram-negative bacteria.

\section{MATERIALS AND METHODS}

\section{Tissue collection}

Tissue samples, including the muscle, kidney, thymus, lung, spleen, liver, bursa of Fabricius, duodenum, jejunum, caeca, and cloaca, were collected from 6 to 8-week-old Pekin duck (Anas platyrhynchos domesticus) at a local farm of Anseong city, Republic of Korea. The samples were washed with phosphate-buffered saline (PBS, $\mathrm{pH}$ 7.4), frozen immediately in liquid nitrogen, and stored at $-80^{\circ} \mathrm{C}$ for future use. The protocol for this experiment was approved by the Institutional Animal Care and Use Committee at Chung-Ang University.

\section{RNA extraction and cDNA synthesis}

The total RNA of tissue samples was extracted with TRIzol Reagent (Invitrogen, Carlsbad, CA, USA) according to the manufacturer's protocol. For cDNA synthesis, $2 \mu \mathrm{g}$ of RNA samples were treated with DNase I (Thermo Scientific, Waltham, MA, USA) and incubated for $30 \mathrm{~min}$ at $37^{\circ} \mathrm{C}$ and then desalted using ethylenediaminetetraacetic acid (Thermo Scientific, USA). Reverse transcription was performed using the RevertAid First Strand cDNA Synthesis Kit (Thermo Scientific, USA) according to the manufacturer's protocols.
Quantitative real-time polymerase chain reaction To analyze the transcripts of duck $L E A P$ - 2 in various organs, the following primers were designed using Primer-BLAST (https://www.ncbi.nlm.nih.gov/tools/primer-blast/): glyceraldehyde-3-phosphate dehydrogenase $(G A P D H)$ forward 5'TGG TGCTGATACGTTGTGGAGTC- $3^{\prime}$ and reverse $5^{\prime}$-AGC TGAGGGGGCGGAGATGA-3', duck-LEAP-2 forward 5'-TG

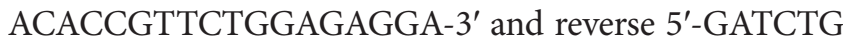
AGGAAGCAGCGGTT-3'. Quantitative real-time polymerase chain reaction (qRT-PCR) was performed using AMPIGENE qPCR Green Mix Lo-ROX (Enzo Life Sciences, Lausen, Switzerland) according to the manufacturer's instructions, using the LightCycler 96 system (Roche, Indianapolis, IN, USA). The duck GAPDH gene was used as a control to normalize for RNA quantity. The relative quantification of gene-specific expression was calculated using the $2^{-\Delta \Delta \mathrm{Ct}}$ method following normalization with the GAPDH gene expression level [19].

\section{Cloning of duck liver-expressed antimicrobial peptide 2}

The primers were designed using DNASTAR (DNASTAR Incorporation, Madison, WI, USA) for amplification of the $L E A P-2$ open reading frame from the predicted duck LEAP-2 cDNA sequence (ENSAPLT00000011688.1). The PCR product was amplified using the specific primers forward 5'-CG GGATCCATGCACTCTTTGAAAGTCATGGC- ${ }^{\prime}$ and reverse 5'-CGGAATTCCTCGGAGGCGGATCTGAG-3' (BamHI and EcoRI restriction enzyme sites are underlined) with a DreamTaq Green PCR Master Mix (2×) (Thermo Scientific, USA). The PCR amplification was achieved under the following condition: a pre-denaturation step at $95^{\circ} \mathrm{C}$ for $5 \mathrm{~min}$, a denaturing step at $94^{\circ} \mathrm{C}$ for $45 \mathrm{~s}$, an annealing step at $55^{\circ} \mathrm{C}$ for $45 \mathrm{~s}$, an extension step at $72^{\circ} \mathrm{C}$ for $45 \mathrm{~s}$ for 35 cycles, and a final extension at $72^{\circ} \mathrm{C}$ for $5 \mathrm{~min}$. The PCR products were purified using the PureLink Quick Gel Extraction Kit (Invitrogen, USA), cloned into the pCR2.1-TOPO vector (Invitrogen, USA), and transformed using Escherichia coli (E. coli) TOP 10 competent cells (Invitrogen, USA) according to the manufacturer's protocol. Through blue-white screening, the positive clones were picked out and then cultured overnight in Luria-Bertani (LB) broth (with $50 \mu \mathrm{g} / \mathrm{mL}$ ampicillin). Plasmids were extracted using NucleoSpin Plasmid (Macherey-Nagel, Düren, North Rhine-Westphalia, Germany) and sequenced at Genotech (Daejeon, Korea).

\section{Recombinant protein expression and purification}

The duck LEAP-2/pCR2.1-TOPO vector was digested with the restriction enzymes EcoRI and BamHI (Promega, Madison, WI, USA). The protein expression vector pET32a (Novagen, Madison, WI, USA) was also digested with the same restriction enzymes. The digested fragments were purified from the agarose gel using the PureLink Quick Gel Extraction Kit (Invitrogen, USA) and were ligated using T4 DNA Ligase (In- 
vitrogen, USA). The ligated vector and insert were transformed into One Shot BL21 (DE3) Chemically Competent E. coli (Invitrogen, USA) and sequenced. Positive clones were incubated at $37^{\circ} \mathrm{C}$ overnight on a shaking incubator at $225 \mathrm{rpm}$ in LB broth with ampicillin $(50 \mu \mathrm{g} / \mathrm{mL})$. The bacteria culture was then induced for recombinant protein expression with $1 \mathrm{mM}$ isopropyl$\beta$-D-thiogalctopyranoside (USB Corporation, Cleveland, $\mathrm{OH}, \mathrm{USA}$ ) for $4 \mathrm{~h}$ at $28^{\circ} \mathrm{C}$, and the bacteria were centrifuged at 5,000 $\times g$ for $15 \mathrm{~min}$. The duck LEAP-2 recombinant protein was extracted with B-PER Bacterial Protein Extraction Reagent (Thermo Scientific, USA) and purified using HisPur Cobalt Resin (Thermo Scientific, USA). Recombinant duck LEAP-2 was eluted using $250 \mathrm{mM}$ imidazole and analyzed by sodium dodecyl sulfate-polyacrylamide gel electrophoresis and western blotting using $6 \times$ His-tag antibody (Thermo Scientific, USA).

\section{Peptide synthesis}

The mature peptide of duck LEAP-2 was synthesized and purified to a $>90 \%$ level using high-performance liquid chromatography by GL Biochem Ltd. (Shanghai, China). Two kinds of duck LEAP-2 peptides were synthesized about this mature peptide sequence. MTPFWRGVSLRPIGASCRDNSECITML CRKNRCFLRSASE; the one is linear type and the other peptide have two disulfide bonds (C17-C28, C23-C33).

\section{Pathogenic bacteria}

The bacterial species used in this experiment included two gram-positive bacteria strains, Listeria monocytogenes (L. monocytogenes) ATCC 19115 and Staphylococcus aureus (S. aureus) ATCC 27664, and four gram-negative bacteria strains, E. coli ATCC 43888, Salmonella enterica serovar Enteritidis YHS 383, Salmonella enterica serovar Choleraesuis YHS 386, and Salmonella enterica serovar Typhimurium ATCC 43174.

\section{Antimicrobial activity assay}

Bacteria were cultured overnight at $37^{\circ} \mathrm{C}$ in $\mathrm{LB}$ broth for E. coli, and in tryptic soy broth for the other bacteria, and suspended to $5.0 \times 10^{5}$ colony-forming units (CFU)/mL in PBS ( $\mathrm{pH} 7.4$ ). To measure antimicrobial activity, $25 \mu \mathrm{L}$ of bacteria were added into 96-well microtiter plates and diluted duck LEAP-2 peptides were dispensed to final concentrations of 50, 100, 150, and $200 \mu \mathrm{g} / \mathrm{mL}$. After $3 \mathrm{~h}$ of incubation at $37^{\circ} \mathrm{C}$, surviving bacteria were counted using a standard colony counting assay according to the following formula: cell survival \% = (treatment $\mathrm{CFU} /$ negative control $\mathrm{CFU}) \times 100$. To determine the killing kinetics, L. monocytogenes and $S$. aureus were cultured overnight in tryptic soy broth and suspended to $5.0 \times 10^{5} \mathrm{CFU} /$ $\mathrm{mL}$ in PBS (pH 7.4). Bacteria were added into 96-well microtiter plates with a final peptide concentration of $200 \mu \mathrm{g} / \mathrm{mL}$. The bacteria and peptide mixture was incubated for $0,30,60$, 90 , and $180 \mathrm{~min}$ at $37^{\circ} \mathrm{C}$. Surviving bacteria were counted using a standard colony counting assay.

\section{Fluorescence microscopic analysis}

E. coli, S. aureus, and L. monocytogenes $\left(5.0 \times 10^{5} \mathrm{CFU} / \mathrm{mL}\right)$ in PBS were incubated with a $200 \mu \mathrm{g} / \mathrm{mL}$ (final concentration) of the disulfide-LEAP-2 peptide for $3 \mathrm{~h}$ at $37^{\circ} \mathrm{C}$. After incubation, the cells were washed with PBS and stained with LIVE/ DEAD BacLight Bacterial Viability Kits (Invitrogen, USA) according to the manufacturer's instructions. In brief, the bacteria were incubated for $15 \mathrm{~min}$ with SYTO9 green fluorescent protein and with propidium iodide in a dark room. The cells were then mounted onto glass slides and examined using EVOS FLoid Cell Imaging Station (Invitrogen, USA).

\section{Bioinformatics analysis}

Purified plasmids were sequenced at Genotech (Korea). To compare the cloned duck LEAP-2 sequence with sequences in GenBank, the data were analyzed using a Nucleotide Basic Local Alignment Search Tool (nBLAST) search (http://www.ncbi. nlm.nih.gov/BLAST/). Protein identification was performed using the Expert Protein Analysis System (https://www.expasy. org/) for determination of the molecular weight and theoretical isoelectric point ( $\mathrm{pI}$ ). Amino acid multiple alignments were generated using CLUSTALW (http://www.genome.jp/tools/ clustalw/) and the MEGA 7 program. The protein structure was predicted by RaptorX (http://raptorx.uchicago.edu/) and FirstGlance in Jmol (http://www.bioinformatics.org/firstglance/fgij/).

\section{Statistical analysis}

The qRT-PCR data were analyzed by one-way analysis of variance followed by Duncan's multiple comparison test using IBM SPSS Statistics 23 software (IBM SPSS Statistics 23 for Windows, Chicago, IL, USA) and antimicrobial activities were analyzed by one-way analysis of variance followed by Dunnett's multiple comparison test. The antimicrobial activity of disulfide and linear LEAP-2 was analyzed by the student's t-test using IBM SPSS Statistics 23 software. The data were expressed as means \pm standard error of the mean and differences were considered statistically significant at $\mathrm{p}<0.05$.

\section{RESULTS}

\section{Bioinformatics analysis}

The duck LEAP-2 peptide was aligned with other avian, fish, amphibian, and mammalian LEAP-2 sequences (Figure 1A). The duck LEAP-2 peptide sequence showed high identity with those of other avian species ( $>85 \%)$, as well as more than $55 \%$ of identity with mammalian sequences, $32 \%$ identity with eel, and $46 \%$ identity with frog (Table 1); overall, there was more than $60 \%$ and $89 \%$ similarity with LEAP-2 of mammalian and avian species, respectively. However, the mature peptide sequence showed more than $90 \%$ similarity with those of other 
A

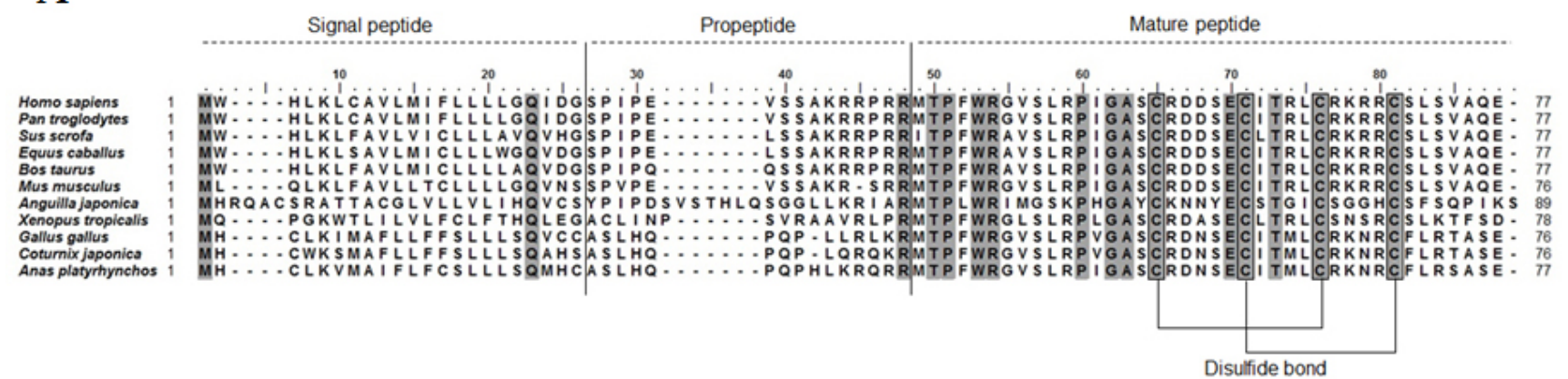

B
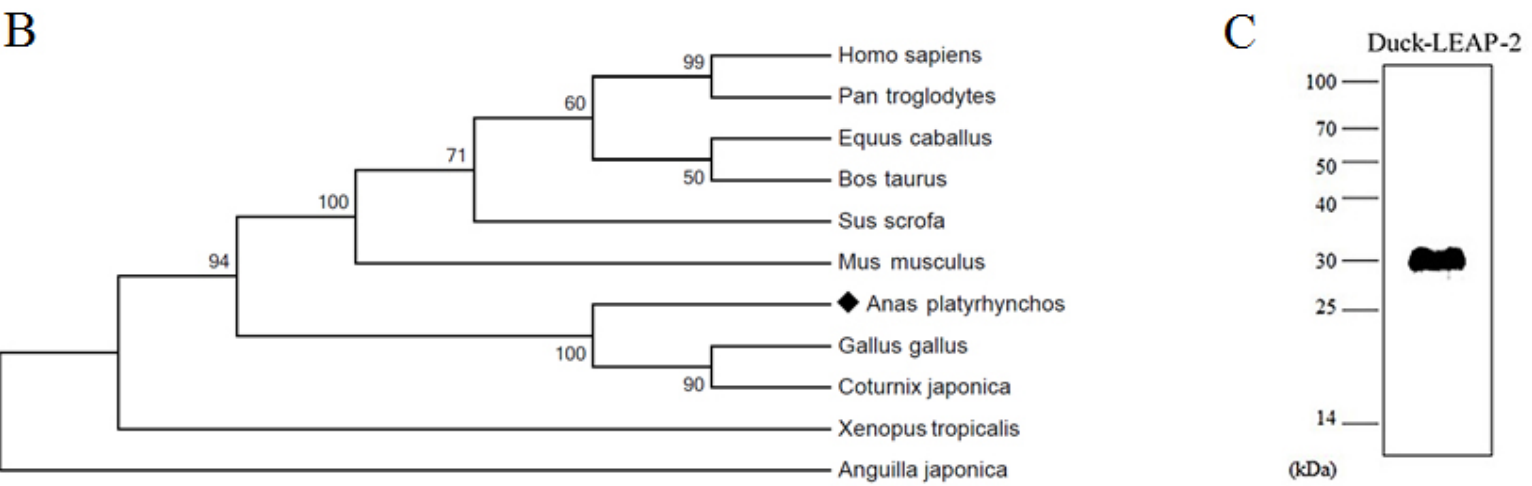

Figure 1. (A) Multiple alignment of the duck LEAP-2 amino acid sequence with several vertebrate LEAP-2 homologs. Conserved sequences are highlighted in gray boxes. (B) Phylogenetic analysis of the complete amino acid sequence of LEAP-2 using the MEGA7.0 program. The values indicate the percentage of trees in which this grouping occurred after bootstrapping. (C) Western blot of recombinant duck LEAP-2 using anti-6x histidine antibody. LEAP-2, liver-expressed antimicrobial peptide 2.

avian, mammalian, and amphibian species. In addition, the mature peptide showed four conserved cysteine residues that consist of disulfide bonds. LEAP-2 sequences could be clearly divided into two groups, with avian species in one group and mammals in the other group. In phylogenetic tree analysis, duck LEAP-2 showed high similarity with chicken LEAP-2 (Figure 1B, Table 1). Therefore, we compared the chicken and duck LEAP-2 gene structures with respect to the exon-intron organization (Figure 2A). Both the duck and chicken LEAP-2 genes were composed of 3 exons and 2 introns; however, the number of base pairs of the exons and introns differed. We also performed structure analysis of duck LEAP-2 protein, and confirmed that duck LEAP-2 is composed of one alpha-helix, two beta-sheets, and two disulfide bonds (Figure 2B).

\section{Production of recombinant duck liver-expressed antimicrobial peptide 2 protein}

We produced recombinant LEAP-2 protein using an E. coli expression system. The molecular weight of duck LEAP-2 was determined to be $8.9 \mathrm{kDa}$ (Signal peptide-propeptide-mature peptide). Because the fusion protein molecular weight in pET32a is $21 \mathrm{kDa}$, the final molecular weight of LEAP-2 was

Table 1. Similarity (upper diagonal) and identity (lower diagonal) of the duck LEAP-2 amino acid sequence with known LEAP-2 sequences of other species (\%)

\begin{tabular}{lcccccccccccc}
\hline & Human & Chimpanzee & Pig & Horse & Cow & Mouse & Eel & Frog & Chicken & Quail & Duck & GenBank Acc No. \\
\hline Human & - & 100 & 89.61 & 94.8 & 92.2 & 88.15 & 46.75 & 61.03 & 64.47 & 60.52 & 62.33 & NP_443203.1 \\
Chimpanzee & 100 & - & 89.61 & 94.8 & 92.2 & 88.15 & 46.75 & 61.03 & 64.47 & 60.52 & 62.33 & XP_001164115 \\
Pig & 85.71 & 85.71 & - & 92.2 & 90.9 & 86.84 & 45.45 & 57.14 & 60.52 & 57.89 & 62.33 & NP_998953.1 \\
Horse & 92.2 & 92.2 & 90.9 & - & 93.5 & 86.84 & 46.75 & 59.74 & 60.52 & 56.57 & 61.03 & XP_003362867.1 \\
Cow & 90.9 & 90.9 & 89.61 & 93.5 & - & 85.52 & 45.45 & 59.74 & 63.15 & 59.21 & 63.63 & NP_776984.1. \\
Mouse & 85.52 & 85.52 & 81.57 & 84.21 & 84.21 & - & 47.36 & 56.57 & 63.15 & 60.52 & 64.47 & NP_694709.1 \\
Eel & 33.76 & 33.76 & 33.76 & 35.06 & 35.06 & 35.52 & - & 42.3 & 44.73 & 43.42 & 41.55 & ALB07167.1 \\
Frog & 49.35 & 49.35 & 48.05 & 46.75 & 46.75 & 46.05 & 29.48 & - & 63.15 & 59.21 & 57.14 & AAl55466.1 \\
Chicken & 57.89 & 57.89 & 52.63 & 55.26 & 57.89 & 57.89 & 34.21 & 51.31 & - & 90.78 & 90.78 & AAS99322.1 \\
Quail & 56.57 & 56.57 & 51.31 & 52.63 & 55.26 & 56.57 & 32.89 & 50 & 90.78 & - & 89.47 & BAU36332.1 \\
Duck & 57.14 & 57.14 & 54.54 & 55.84 & 58.44 & 59.21 & 32.46 & 46.75 & 85.52 & 85.52 & - & ENSAPLP00000010969.1 \\
\hline
\end{tabular}

LEAP-2, liver-expressed antimicrobial peptide 2. 
A
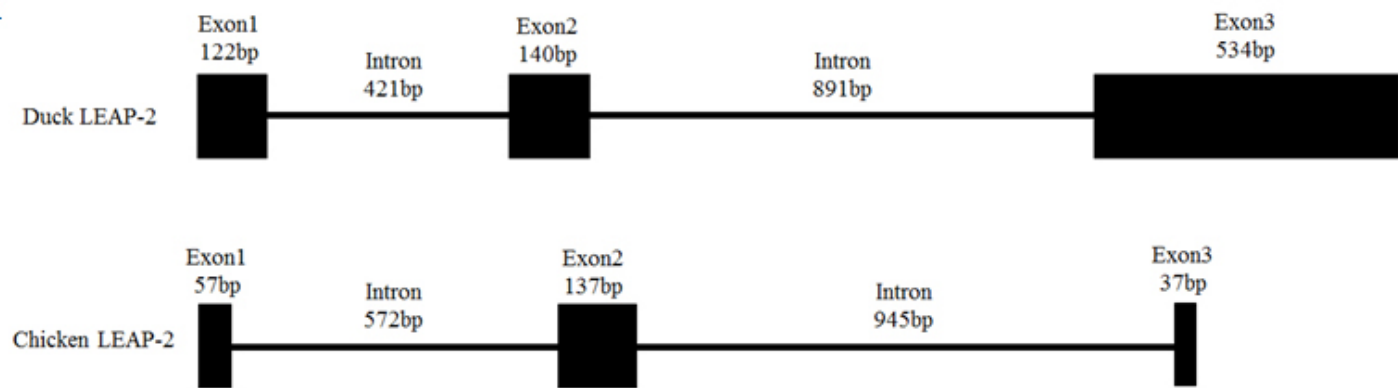

$\mathrm{B}$

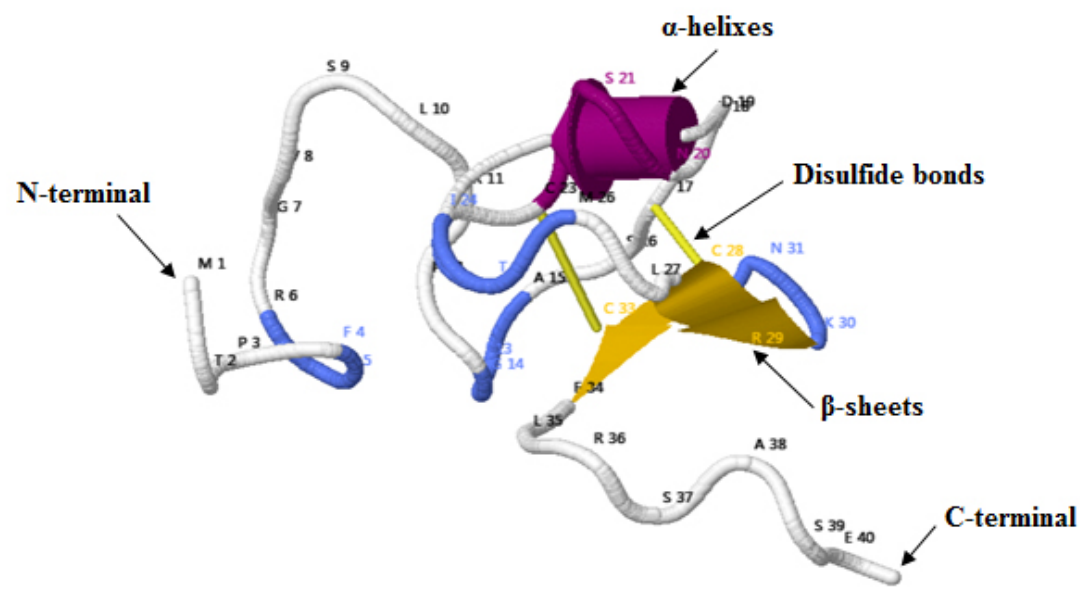

Figure 2. (A) Comparison of the exon-intron compositions of the duck LEAP-2 and chicken LEAP-2 gene structures. The black rectangles indicate exons and introns in line. (B) Structural analysis of duck LEAP-2 protein. The letters on the line are the amino acid abbreviations, and the numbers next to the abbreviations indicate the amino acid positions in the protein. Alpha helices are shown as red "rockets", beta strands are shown as yellow planks, blue lines indicate turns, and yellow sticks indicate disulfide bonds. LEAP-2, liver-expressed antimicrobial peptide 2.

approximately $30 \mathrm{kDa}$. We also confirmed the expression of recombinant LEAP-2 by western blotting using $6 \times$ His-tag antibody (Figure 1C).

Tissue expression profiles of duck liver-expressed antimicrobial peptide 2

We measured the mRNA level of duck LEAP-2 in the muscle, kidney, thymus, lung, spleen, liver, bursa of Fabricius, duodenum, jejunum, caeca, and cloaca by real-time PCR. The LEAP-2 mRNA transcript level was the highest in the liver with duodenum next, and then followed by lung, spleen, bursa and jejunum and was the lowest in the muscle (Figure 3).

\section{Antimicrobial activity of synthetic duck liver-expressed}

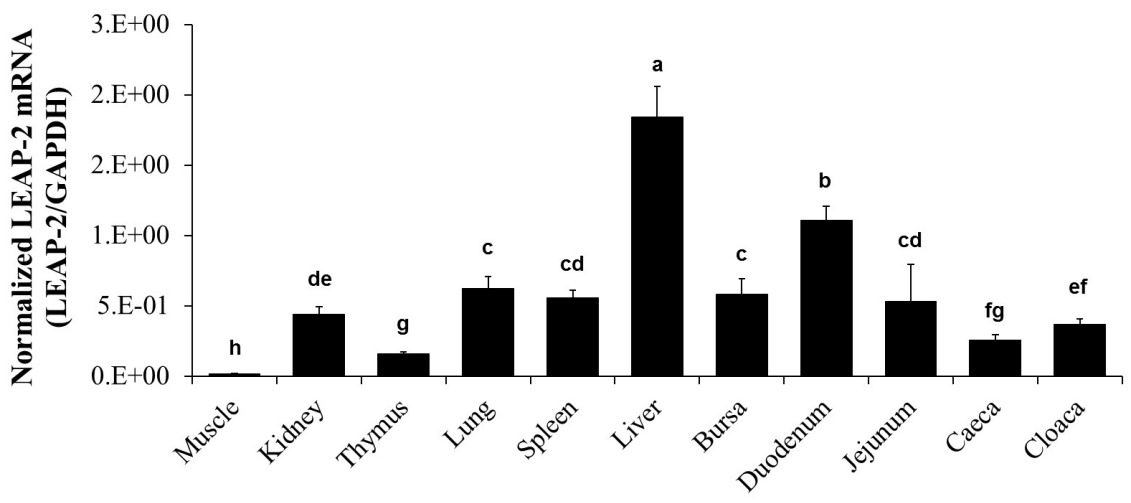

Figure 3. Duck LEAP-2 mRNA expression levels in various organs. Duck LEAP-2 mRNA transcript levels were determined in the muscle, kidney, thymus, lung, spleen, liver, bursa of Fabricius, duodenum, jejunum, caeca, and cloaca by real-time polymerase chain reaction. The data are presented as normalized mRNA levels to GAPDH. LEAP-2, liver-expressed antimicrobial peptide 2; GAPDH, glyceraldehyde-3-phosphate dehydrogenase. 


\section{antimicrobial peptide 2 peptide}

We synthesized two peptides, a disulfide bond LEAP-2 and linear LEAP-2 peptide, to measure the LEAP-2 antimicrobial activity. We treated the peptides with gram positive and gram negative bacteria for 3 hours by diversifying the concentration. LEAP-2 showed a significant killing effect for the gram positive bacteria $S$. aureus and L. monocytogenes (Figure 4A, 4B). In particular, the disulfide LEAP-2 peptide killed most of the S. aureus and L. monocytogenes at $50 \mu \mathrm{g} / \mathrm{mL}$. Gram-negative bacteria were also killed by LEAP-2, but the efficiency was lower than that against gram-positive bacteria (Figure 4C-4F).
As shown in Figure 4C, linear LEAP-2 killed 60\% of E. coli at $200 \mu \mathrm{g} / \mathrm{mL}$. However, disulfide LEAP-2 killed more than $90 \%$ of $E$. coli at $50 \mu \mathrm{g} / \mathrm{mL}$. The two peptides showed similar patterns against $S$. Choleraesuis, in which more than $70 \%$ of the cells were inhibited at $200 \mu \mathrm{g} / \mathrm{mL}$. Linear LEAP-2 showed a $50 \%$ killing effect for $S$. Typhimurium, whereas disulfide LEAP-2 inhibited $90 \%$ of the cell proliferation at $200 \mu \mathrm{g} / \mathrm{mL}$. $S$. Enteritidis was also inhibited by $60 \%$ with the disulfide LEAP-2, although it was not inhibited by linear LEAP-2. Taken together, LEAP-2 showed more powerful antibacterial activity to gram positive bacteria than gram negative bacteria. Also,
A
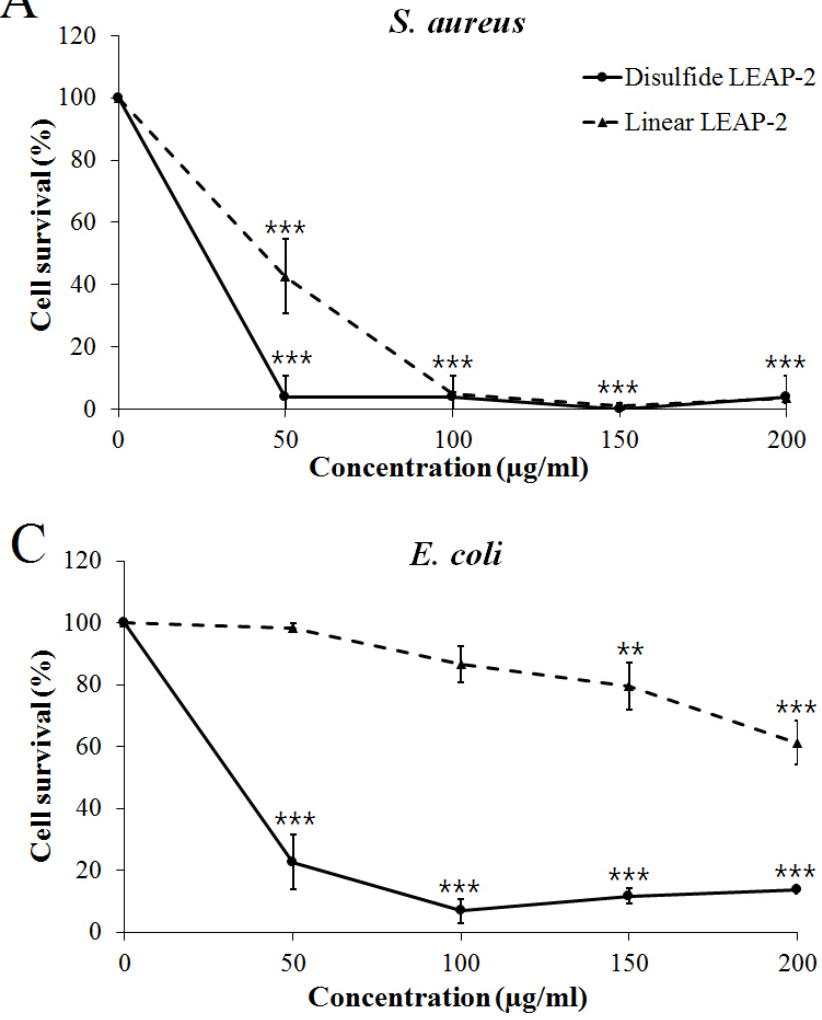

$\mathrm{E}$

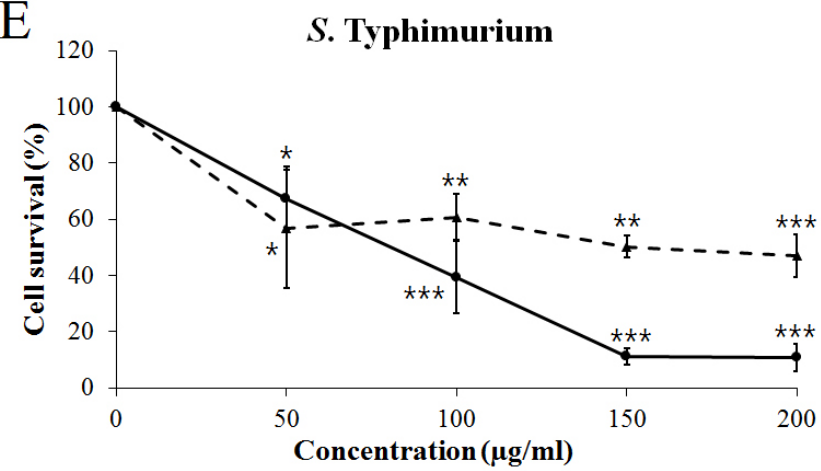

$\mathrm{B}$

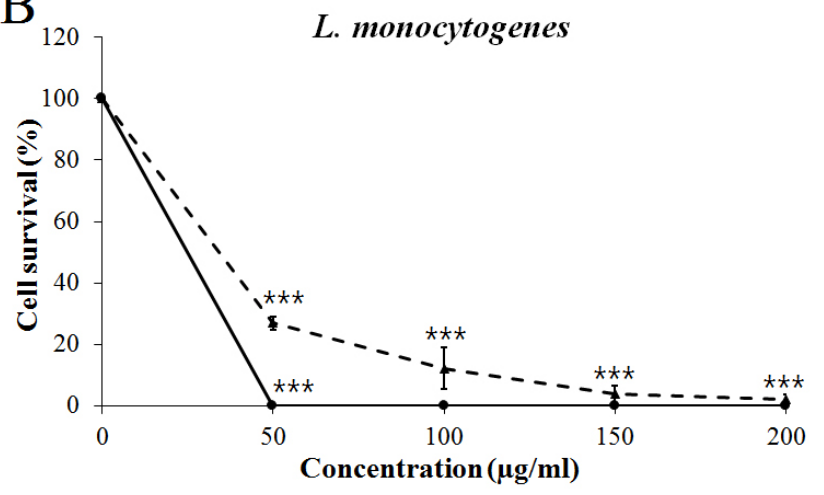

$\mathrm{D}$

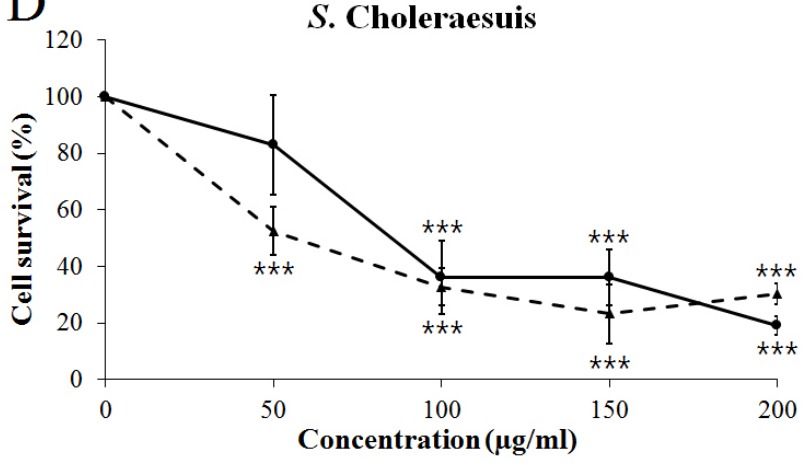

$\mathrm{F}$

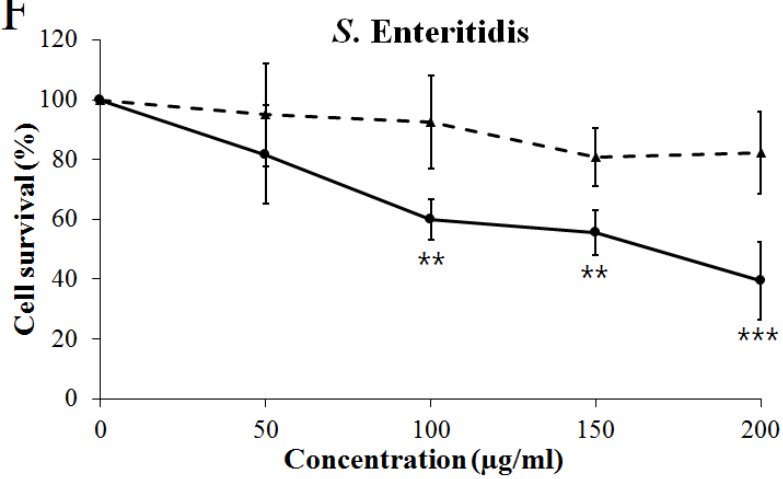

Figure 4. Antibacterial activity of synthetic duck LEAP-2 peptides. The pathogens were treated with linear LEAP-2 and disulfide LEAP-2 peptides and incubated for $3 \mathrm{~h}$ at $37^{\circ} \mathrm{C}$. (A) Staphylococcus aureus ATCC 276674, (B) Listeria monocytogenes ATCC 19115, (C) Escherichia coli ATCC 43888, (D) Salmonella enterica serovar Choleraesuis YHS 386, (E) Salmonella enterica serovar Typhimurium ATCC 43174, (F) Salmonella enterica serovar Enteritidis YHS 383. The solid line is the disulfide LEAP-2 peptide and the dotted line is the linear LEAP-2 peptide. Cell survival (\%) was calculated as (treatment CFU/negative control CFU) $\times 100$. LEAP-2, liver-expressed antimicrobial peptide 2; $\mathrm{CFU}$, colony-forming units. Each data point is the mean \pm standard error of the mean $\left({ }^{*} p<0.05,{ }^{* *} p<0.01\right.$, and $\left.{ }^{* * *} p<0.001\right)$. 
disulfide LEAP-2 showed better killing effect than linear LEAP2 peptide by showing a stronger bactericidal effect except for $S$. Cholerasuis.

\section{Killing kinetics of synthetic duck liver-expressed antimicrobial peptide 2}

L. monocytogenes and S. aureus were used to determine the killing kinetics of duck LEAP-2 over time. Both the disulfide LEAP-2 and linear LEAP-2 peptides effectively killed all of the L. monocytogenes in only $30 \mathrm{~min}$ (Figure 5A). In addition, both disulfide LEAP-2 and linear LEAP-2 killed S. aureus gradually over time, with disulfide LEAP-2 showing a much better effect (Figure 5B).

\section{Fluorescence microscopic analysis for live/dead} pathogens

To visualize the killing effect of duck LEAP-2, a live/dead staining method was carried out. L. monocytogenes, S. aureus, and E. coli were incubated with the disulfide peptide for $3 \mathrm{~h}$ before staining. Many of the cells in the peptide treatment group showed red fluorescence, indicating that most of the cells were dead (Figure 6), whereas the cells of the control group (no peptide treatment) showed only green fluorescence, indicating that most of the cells were alive.

\section{DISCUSSION}

AMPs of animals are potentially powerful antibiotics substitutes. LEAP- 2 is a generally well-known AMPs in mammals, avian, and fish that has bactericidal effects $[12,15,17,18,20]$.

In general, AMPs have to bind to the bacterial cell membrane to exert their effects [6]. Because most bacterial cell membranes have a net negative charge due to the presence of anionic phospholipids, lipopolysaccharide on the surface of gram-negative bacteria, and teichoic acids on the surface of gram-positive bacteria, the cationic AMPs can bind to the cell membrane. After binding to the cell surface, AMPs create a pore such as a toroidal pore, carpet, or barrel stave [21], which then ruptures the bacterial cell to ultimately kill the bacteria [22].

In the current study, we first identified duck LEAP-2 and characterized its antimicrobial function against pathogens. In
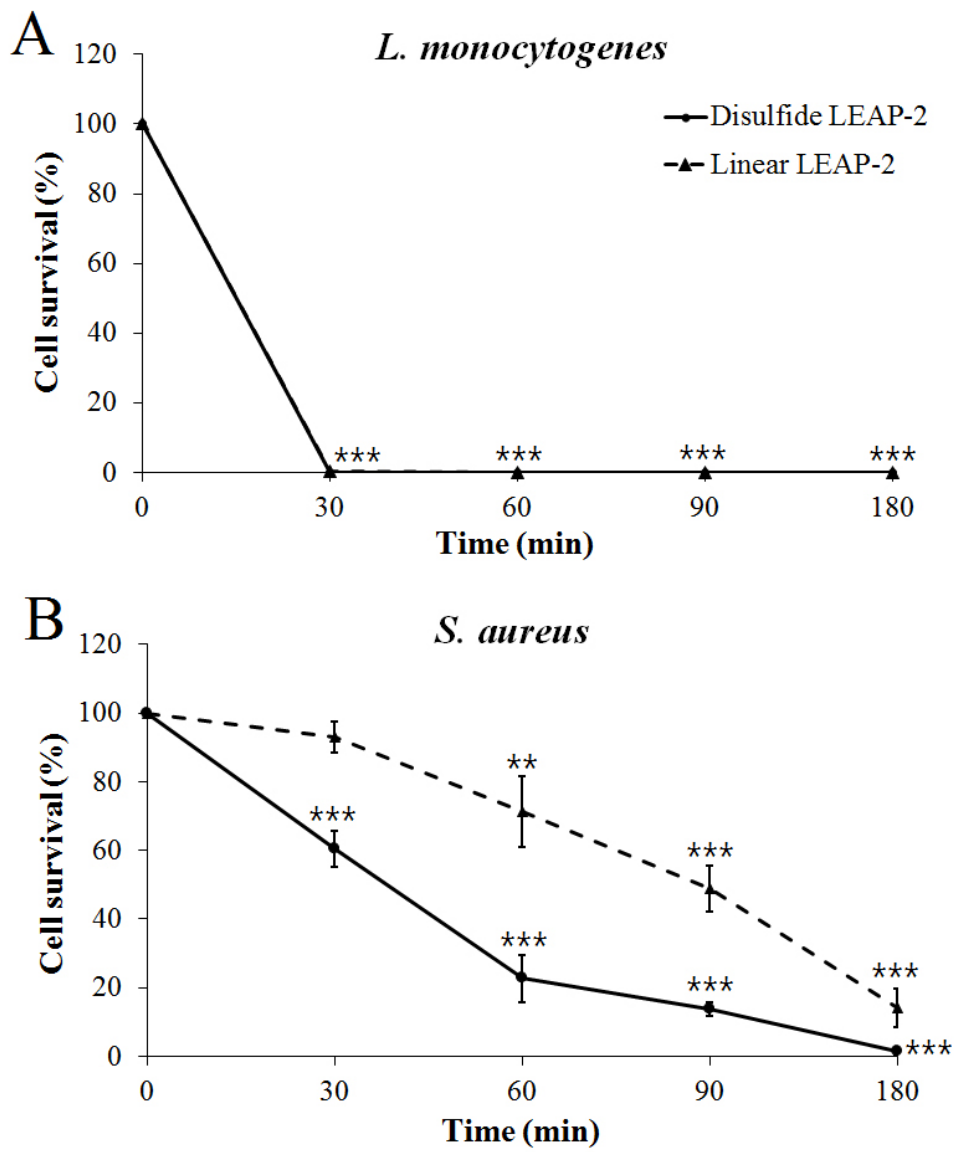

Figure 5. Killing kinetics of synthetic duck LEAP-2 against gram-positive bacteria. (A) Listeria monocytogenes ATCC 19115, (B) Staphylococcus aureus ATCC 276674. The pathogens were treated with linear LEAP-2 and disulfide LEAP-2 and incubated for 30,60,90, and 180 min at $37^{\circ} \mathrm{C}$. The solid line is the disulfide LEAP-2 peptide and the dotted line is linear LEAP-2 peptide. Cell survival (\%) was calculated as (treatment CFU/negative control CFU) $\times 100$. LEAP-2, liver-expressed antimicrobial peptide 2; CFU, colony-forming units. Each data point is the mean \pm standard error of the mean $\left({ }^{*} p<0.05,{ }^{* *} p<0.01\right.$, and $\left.{ }^{* * *} p<0.001\right)$. 

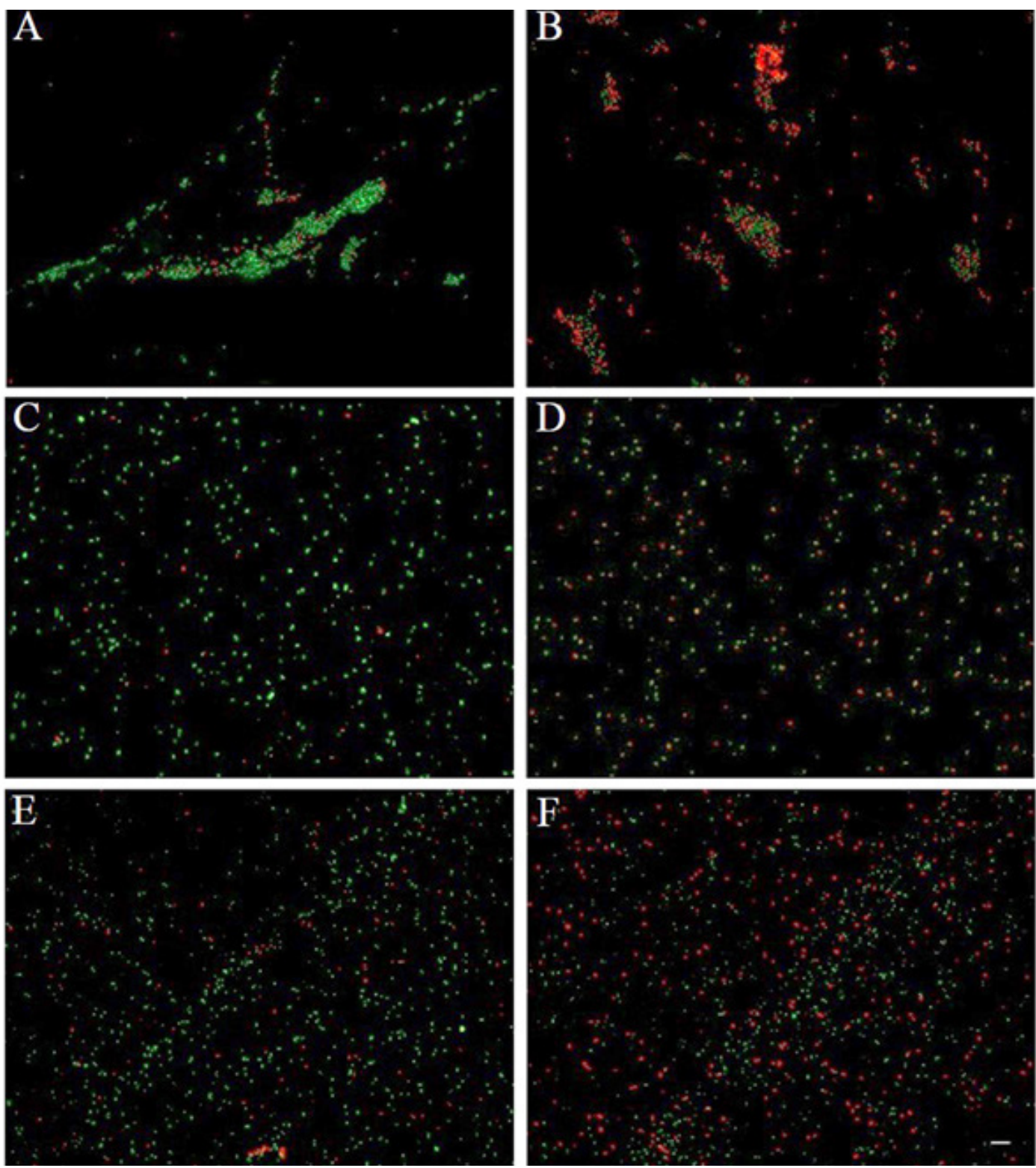

Figure 6. Fluorescence microscopy analysis of disrupted bacteria. Bacteria were treated with disulfide liver-expressed antimicrobial peptide 2 peptide $(200 \mu \mathrm{g} / \mathrm{mL})$ and incubated for $3 \mathrm{~h}$ at $37^{\circ} \mathrm{C}$. Bacteria were stained with a LIVE/DEAD BacLight Bacterial Viability Kit. (A) Control Escherichia coli, (B) treated Escherichia coli, (C) control Staphylococcus aureus, (D) treated Staphylococcus aureus, (E) control Listeria monocytogenes, (F) treated Listeria monocytogenes. Green fluorescence indicates live bacteria and red fluorescence indicates dead bacteria with damaged membranes. Scale bar $=7 \mu \mathrm{m}$.

addition, we predicted the duck LEAP-2 gene structure as a signal peptide, pro-peptide, and mature peptide based on the LEAP-2 sequences of mammals and other avian species (Figure 1). Duck LEAP-2 shows conserved mature peptide sequences and two disulfide bonds similar to those of other species based on alignment of the protein sequence and phylogenetic analysis. In general, disulfide bonds play an important role in the folding and stability of certain proteins [23-26]. A previous study also showed that conserved disulfide bridges in avian- $\beta$-defensin- 12 are essential for the chemotactic property and maximum antimicrobial activity [27]. Therefore, we could predict that these disulfide bonds are an important component for the bactericidal effect of duck LEAP-2.

In mammals, human, pig, and horse, LEAP-2 mRNA has been shown to be expressed mainly in the liver, kidney, and intestine $[12,14,28,29]$. In addition, chicken LEAP-2 mRNA was reported to be mainly expressed in the liver, kidney, small intestine, and lung $[18,30,31]$. As expected, we found that the duck LEAP-2 expression levels were the highest in the liver, with the remaining distribution corresponding to some extent with that of the chicken (Figure 3).

To confirm the importance of the disulfide bond in the bactericidal effect, we synthesized two kinds of peptides, linear and disulfide. The disulfide LEAP-2 peptide showed a more 
powerful bactericidal effect than the linear form except against $S$. Choleraesuis. The AMP should have contact with bacterial cell membrane to show antibacterial activity. So, the peptide structure will be important for affinity. Originally, duck LEAP2 has two disulfide bonds in mature peptide, so we think that original structure of LEAP-2 has more potent killing effect. Considering the mode of action of most AMPs, the conserved disulfide bonds in LEAP-2 likely play a crucial role in the stability of the peptide.

We tested the duck LEAP-2 recombinant protein against various bacterial strains (data not shown). Unfortunately, we did not observe a substantial bacterial killing effect. Prokaryotic protein expression differs from the eukaryotic protein expression system, and due to translational modifications such as protein glycosylation, some recombinant proteins expressed in prokaryotes might not be suitable for functional recombinant protein production [32]. Therefore, we suggest that the large size of the fusion protein affected the LEAP-2 structure and function [33].

With live/dead staining, we demonstrated that the bacteria cell membranes were disrupted by LEAP-2 peptides (Figure 6). SYTO9 green fluorescent protein stains the nucleic acids of all bacteria with intact membranes, whereas propidium iodide can only penetrate bacteria cells with damaged membranes, causing a reduction in the SYTO9 fluorescence when both dyes are present. Therefore, we could confirm that the mode of action of LEAP-2 is membrane destruction. The main mechanism for membrane destruction is the binding of AMPs to the bacterial cell envelope. As mentioned above, lipoteichoic acid and lipopolysaccharide confer the bacterial cell envelope with a negative charge, and most AMPs have a positive charge [2-4]; thus, this mode of action suggests that LEAP-2 also has an overall positive charge and results in cell envelope disruption.

The net charge of a protein is affected by its own $\mathrm{pI}$ and the $\mathrm{pH}$ of the surrounding environment. When present at a $\mathrm{pH}$ lower than their pI, proteins obtain a net positive charge. In contrast, when present at a $\mathrm{pH}$ above their $\mathrm{pI}$, they obtain a net negative charge [34]. The pI of the mature duck LEAP-2 peptide was determined to be 9.11. Because the physiological $\mathrm{pH}$ such as that in the gastrointestinal tract and blood is lower than 9.11, duck LEAP-2 would carry a net positive charge in a physiological environment. Therefore, cationic LEAP- 2 would bind to the negatively charged residues of the bacterial cell wall or membrane. After LEAP-2 binds to the bacteria, it will disrupt the cell envelope by making pores, and consequently cause the leakage of cell contents and cell death.

Taken together, our study identified duck LEAP-2 and demonstrated its antimicrobial activity against both gram-positive and gram-negative bacteria. In particular, we identified the importance of the disulfide bond for a powerful killing effect by disrupting the bacterial cell envelope. Therefore, duck LEAP-
2 may be used as an effective AMPs to substitute for antibiotics and as a novel disease control agent in the future.

\section{CONFLICT OF INTEREST}

We certify that there is no conflict of interest with any financial organization regarding the material discussed in the manuscript.

\section{ACKNOWLEDGMENTS}

This work was supported by a National Research Foundation grant (NRF-2015R1D1A1A09059345) of the Republic of Korea and NIFA grant (\#2017-6701526793) of USDA.

\section{REFERENCES}

1. Kim WH, Lillehoj HS, Gay CG. Using genomics to identify novel antimicrobials. Rev Sci Tech 2016;35:95-103.

2. Dürr UH, Sudheendra U, Ramamoorthy A. LL-37, the only human member of the cathelicidin family of antimicrobial peptides. Biochim Biophys Acta 2006;1758:1408-25.

3. Papagianni M. Ribosomally synthesized peptides with antimicrobial properties: biosynthesis, structure, function, and applications. Biotechnol Adv 2003;21:465-99.

4. Sitaram N, Nagaraj R. Host-defense antimicrobial peptides: importance of structure for activity. Curr Pharm Des 2002;8: 727-42.

5. Lee SH, Lillehoj HS, Tuo W, Murphy CA, Hong YH, Lillehoj EP. Parasiticidal activity of a novel synthetic peptide from the core alpha-helical region of NK-lysin. Vet Parasitol 2013;197: 113-21.

6. Reddy K, Yedery R, Aranha C. Antimicrobial peptides: premises and promises. Int J Antimicrob Agents 2004;24:536-47.

7. Bals R, Wilson J. Cathelicidins-a family of multifunctional antimicrobial peptides. Cell Mol Life Sci 2003;60:711-20.

8. Beisswenger $C$, Bals R. Antimicrobial peptides in lung inflammation. Chem Immunol Allergy 2005;86:55-71.

9. Engström Y. Induction and regulation of antimicrobial peptides in Drosophila. Dev Comp Immunol 1999;23:345-58.

10. Hetru C, Troler L, Hoffmann JA. Drosxophila melanogaster antimicrobial defense. J Infect Dis 2003;187:S327-S34.

11.Lehrer RI, Ganz T. Antimicrobial peptides in mammalian and insect host defence. Curr Opin Immunol 1999;11:23-7.

12. Krause A, Sillard R, Kleemeier B, et al. Isolation and biochemical characterization of LEAP-2, a novel blood peptide expressed in the liver. Protein Sci 2003;12:143-52.

13.Lynn DJ, Lloyd AT, O’Farrelly C. In silico identification of components of the Toll-like receptor (TLR) signaling pathway in clustered chicken expressed sequence tags (ESTs). Vet Immunol Immunopathol 2003;93:177-84.

14.Sang Y, Ramanathan B, Minton JE, Ross CR, Blecha F. Porcine 
liver-expressed antimicrobial peptides, hepcidin and LEAP2: cloning and induction by bacterial infection. Dev Comp Immunol 2006;30:357-66.

15. Townes CL, Michailidis G, Hall J. The interaction of the antimicrobial peptide CLEAP-2 and the bacterial membrane. Biochem Biophys Res Commun 2009;387:500-3.

16. Harwig SS, Waring A, Yang HJ, Cho Y, Tan L, Lehrer RI. Intramolecular disulfide bonds enhance the antimicrobial and lytic activities of protegrins at physiological sodium chloride concentrations. Eur J Biochem 1996;240:352-7.

17. Ishige T, Hara H, Hirano T, Kono T, Hanzawa K. Characterization and expression of non-polymorphic liver expressed antimicrobial peptide 2: LEAP-2 in the Japanese quail, Coturnix japonica. Anim Sci J 2016;87:1182-7.

18. Townes CL, Michailidis G, Nile CJ, Hall J. Induction of cationic chicken liver-expressed antimicrobial peptide 2 in response to Salmonella enterica infection. Infect Immun 2004;72:6987-93.

19.Livak KJ, Schmittgen TD. Analysis of relative gene expression data using real-time quantitative PCR and the $2^{-\triangle \Delta C T}$ method. Methods 2001;25:402-8.

20.Zhang Y-A, Zou J, Chang C-I, Secombes CJ. Discovery and characterization of two types of liver-expressed antimicrobial peptide 2 (LEAP-2) genes in rainbow trout. Vet Immunol Immunopathol 2004;101:259-69.

21. Brogden KA. Antimicrobial peptides: pore formers or metabolic inhibitors in bacteria? Nat Rev Microbiol 2005;3:238-50.

22. Kalfa V, Jia H, Kunkle R, McCray P, Tack B, Brogden K. Congeners of SMAP29 kill ovine pathogens and induce ultrastructural damage in bacterial cells. Antimicrob Agents Chemother 2001;45:3256-61.

23. Betz SF. Disulfide bonds and the stability of globular proteins. Protein Sci 1993;2:1551-8.
24. Creighton TE. Disulphide bonds and protein stability. BioEssays 1988;8:57-63.

25.Sevier CS, Kaiser CA. Formation and transfer of disulphide bonds in living cells. Nat Rev Mol Cell Biol 2002;3:836-47.

26. Wanniarachchi YA, Kaczmarek P, Wan A, Nolan EM. Human defensin 5 disulfide array mutants: disulfide bond deletion attenuates antibacterial activity against Staphylococcus aureus. Biochemistry 2011;50:8005-17.

27. Yang M, Zhang C, Zhang MZ, Zhang S. Novel synthetic analogues of avian $\beta$-defensin-12: the role of charge, hydrophobicity, and disulfide bridges in biological functions. BMC Microbiol 2017; $17: 43$.

28. Hocquellet A, Odaert B, Cabanne C, et al. Structure-activity relationship of human liver-expressed antimicrobial peptide 2. Peptides 2010;31:58-66.

29.Etmektedir, SİI. The increase in LEAP-2 mRNA suggests a synergistic probiotics-doxycycline interaction in chickens. Turk J Immunol 2017;5:5-12.

30.Lynn DJ, Higgs R, Gaines S, et al. Bioinformatic discovery and initial characterisation of nine novel antimicrobial peptide genes in the chicken. Immunogenetics 2004;56:170-7.

31. Michailidis G. Expression of chicken LEAP-2 in the reproductive organs and embryos and in response to Salmonella enterica infection. Vet Res Commun 2010;34:459-71.

32. Parachin NS, Mulder KC, Viana AAB, Dias SC, Franco OL. Expression systems for heterologous production of antimicrobial peptides. Peptides 2012;38:446-56.

33.Li Y. Recombinant production of antimicrobial peptides in Escherichia coli: a review. Protein Expr Purif 2011;80:260-7.

34. Patrickios CS, Yamasaki EN. Polypeptide amino acid composition and isoelectric point ii. comparison between experiment and theory. Anal Biochem 1995;231:82-91. 